\title{
siRNA targeting YAP gene inhibits gastric carcinoma growth and tumor metastasis in SCID mice
}

\author{
ZHOU ZHOU ${ }^{1}$, JIN-SHUI ZHU ${ }^{2}$, CAI-PING GAO ${ }^{1}$, LIANG-PING LI ${ }^{1}$, CHAO ZHOU ${ }^{1}$, \\ HAN WANG ${ }^{1}$ and XIAO-GANG LIU ${ }^{1}$ \\ ${ }^{1}$ Department of Gastroenterology, Sichuan Provincial People's Hospital, Chengdu, Sichuan 610072; \\ ${ }^{2}$ Department of Gastroenterology, Shanghai Jiao Tong University Affiliated \\ Sixth People's Hospital, Shanghai 200233, P.R. China
}

Received December 27, 2014; Accepted February 11, 2016

DOI: $10.3892 / \mathrm{ol} .2016 .4319$

\begin{abstract}
Yes-associated protein (YAP) is constitutively activated in numerous types of cancer, including gastric carcinoma. The aim of the present study was to investigate the effects of YAP silencing on proliferation, apoptosis, metastasis and angiogenesis in a gastric orthotopic implantation cancer model of severe combined immunodeficiency mice. Small-hairpin RNA (shRNA) targeting the YAP gene was employed to inhibit YAP expression. SGC7901 cells transfected with YAP shRNA demonstrated significantly decreased gastric cancer growth and metastasis in the orthotopic implantation mouse model. Silencing of YAP additionally promoted tumor cell apoptosis, and inhibited tumor cell proliferation and angiogenesis. Notably, YAP shRNA also downregulated the expression of TEA domain family member 1, cyclinD1, vascular endothelial growth factor and fibroblast growth factor-2. The results of the present study suggested that YAP may have a significant role in the proliferation, metastasis and angiogenesis of gastric cancer. RNA interference-mediated silencing of YAP may provide an opportunity to develop a novel treatment strategy for gastric cancer.
\end{abstract}

\section{Introduction}

Gastric cancer is the fourth most common cancer in the world, with an estimated 988,000 cases in 2008 worldwide and an estimated 736,000 mortalities occurring as a result of the disease (1). More than $70 \%$ of cases occur in developing countries (1). It is characterized by high incidence, frequent metastasis, high mortality and relative unresponsiveness to

Correspondence to: Professor Xiao-Gang Liu, Department of Gastroenterology, Sichuan Provincial People's Hospital, 32 West Second Section First Ring Road, Chengdu, Sichuan 610072, P.R. China

E-mail:xgliu2003@aliyun.com

Key words: yes-associated protein gene, gastric carcinoma, RNA interference, orthotopic implantation mouse model standard oncological therapies, including radiotherapy and chemotherapy (2). The overall five-year survival rate is $<40 \%$, due to relapse and metastasis (2).

Yes-associated protein (YAP) is a $65 \mathrm{kD}$ proline-rich phosphoprotein, which is located at locus 11q22 (3). A previous study reported evidence that YAP may have oncogenic functions, and an increasing amount of literature has associated elevated YAP expression with malignant tumors (4). In MCF-10A cells, YAP gene overexpression induces epithelial-to-mesenchymal transition, which is a characteristic of malignant cell transformation (5). YAP additionally combines with myc to promote growth of tumors in mice (6). Notably, liver-specific YAP gene overexpression leads to hepatic carcinoma in transgenic mice (7). YAP is a member of the Hippo signaling cascade, which consists of protein kinases and regulatory proteins, including merlin, the mammalian Hippo homolog, salvador/WW45 and Lats1/2. When activated the Hippo pathway antagonizes YAP via phosphorylation and binding to $14-3-3$ protein, resulting in cytoplasmic sequestration (8).

RNA interference (RNAi) is a novel type of genetic tool that is able to mediate posttranscriptional sequence-specific gene silencing (9). The authors of the present study established the YAP knockdown SGC7901 gastric cancer cell line using lentivirus-mediated small hairpin (sh)RNA and observed that silencing of YAP led to effective inhibition of tumor cell growth and invasive ability in vitro $(10,11)$. To identify the effect of YAP silencing in vivo and explore its mechanism, the present study performed animal experiments using a mouse carcinoma model.

In the present study, it was demonstrated that inhibition of YAP expression results in the reversal of a number of properties associated with the malignant phenotype, including proliferation and metastasis in vivo. The results of the present study suggest that YAP may be a potential therapeutic target for the treatment of gastric cancer.

\section{Materials and methods}

Materials. SGC7901 cells were purchased from the Shanghai Institute of Biochemistry and Cell Biology (Shanghai, China). TRIzol ${ }^{\circledR}$ Reagent was purchased from Thermo Fisher Scientific, Inc. (Waltham, MA, USA). The lentivirus system against YAP 
gene consisting of pGC-LV, pHelper 1.0 and pHelper 2.0 was obtained from GeneChem Co., Ltd. (Shanghai, China) and was constructed as previously described (11). The YAP rabbit anti-human polyclonal antibody (sc-15407), the cluster of differentiation (CD)31 rabbit anti-human polyclonal antibody (sc-8306), the vascular endothelial growth factor receptor (VEGF) rabbit anti-human polyclonal antibody (sc-152), the cyclinD1 rabbit anti-human polyclonal antibody (sc-753), the Ki-67 goat anti-mouse polyclonal antibody (sc-7846), the fibroblast growth factor (FGF)-2 rabbit anti-human polyclonal antibody (sc-79), the cyclinA rabbit anti-human polyclonal antibody (sc-751), the cyclinE rabbit anti-rat polyclonal antibody (sc-481) and the glyceraldehyde-3-phosphate dehydrogenase (GAPDH) rabbit anti-rat polyclonal antibody (sc-25778) were purchased from Santa Cruz Biotechnology, Inc. (Dallas, TX, USA). The TEA domain family member 1 (TEAD) rabbit anti-human polyclonal antibody (13283-1-AP) was purchased from ProteinTech Group, Inc., (Chicago, IL, USA). ECL Western Blotting Substrate (32106) was purchased from Thermo Fisher Scientific, Inc.. TUNEL assay kit (KGA700) was purchased from Nanjing KeyGen Biotech Co., Ltd. (Nanjing, China). All primers were synthesized by the Shanghai Sangon Biological Engineering Technology and Service Co., Ltd. (Shanghai, China). RPMI-1640 medium was obtained from Gibco (Thermo Fisher Scientific, Inc.) and fetal bovine serum was purchased from Zhejiang Tianhang Biotechnology Co., Ltd. (Hangzhou, China).

Animals. Female severe combined immunodeficiency mice (SCID; 6 weeks old) were purchased from the Shanghai Laboratory Animal Center of the Chinese Academy of Sciences (Shanghai, China). All 5 mice were kept in laminar flow cabinets under specific pathogen-free conditions, with food and water ad libitum (humidity 30-50\%, temperature $20-22^{\circ} \mathrm{C}$ and a 12 -h light-dark cycle). The mice were split into groups for the use of the study at random. The use of animals in this study was approved by Sichuan Medical Experimental Animal Care Committee (Chengdu, China).

Preparation of SGC7901 cells with YAP gene expression stably inhibited. In our previous study (11), we successfully synthesized a lentiviral vector small hairpin (sh)RNA (5'-CTCAGGATGGAGAAATTTA-3') targeting the YAP gene, which efficiently inhibited YAP expression at the messenger (m)RNA and protein level in vitro. A control vector carrying a sequence (5'-TTCTCCGAACGTGTC ACGT-3') unrelated to the human gene was used as a negative control. Subsequently, these vectors were transfected into SGC7901 cells separately. During transfection, cells were seeded in a 24 well plate with confluence reaching $80 \%$ prior to transfection. A total of $4 \mu 1$ Lipofectamine $2000^{\circledR}$ (11668-027; Thermo Fisher Scientific, Inc.) and $5 \mu \mathrm{g}$ plasmid DNA were mixed in Opti-MEM medium (31985-088; Thermo Fisher Scientific, Inc.) and added to the cells of each well according to manufacturer's protocol. Cells were selected for stable expression by culturing in puromycin medium (Thermo Fisher Scientific, Inc.) for 6 weeks. A total of 3 experimental groups were designed as follows: The YAP shRNA vector-transfected cells (YAP-shRNA group), negative control vector-transfected cells (NC group) and untransfected cells (CON group).
Gastric orthotopic implantation tumor model. SCID mice were injected subcutaneously into the dorsal scapula region with $1 \times 10^{6}$ SGC7901 cells from one of the three groups (YAP-shRNA, NC or CON). Once xenografts were established, the tumors were removed and washed twice with phosphate-buffered saline (PBS). Cancer tissue was divided into small pieces $\left(\sim 1 \mathrm{~mm}^{3}\right)$ and gastric surgical orthotopic implantation of the tumor was performed. Briefly, following anesthetizing of the mice with $2.5 \%$ Avertin (Sigma-Aldrich, St. Louis, MO, USA), the stomach was exteriorized. The small sections of cancer tissue were sewn onto the gastric wall and the laparotomy was closed. When the animals become moribund during the observation period ( 2 months later), the mice were sacrificed via cervical dislocation., organs were excised, and metastases were determined by observation and further confirmation by hematoxylin and eosin staining.

Reverse transcription- quantitative polymerase chain reaction $(R T-q P C R)$ assay. RT-qPCR was used to quantitatively measure the mRNA expression level. Total RNA from the mouse orthotopic gastric tumor was extracted by using TRIzol reagent according to the manufacturer's protocol. During RNA extraction, DNase I ( $1 \mathrm{u} / \mu \mathrm{g}$; Thermo Fisher Scientific Inc.) was added to the sample following the manufacturer's protocol. Subsequently, $2 \mu \mathrm{g}$ of total RNA was reverse-transcribed with Moloney Murine Leukemia Virus reverse transcriptase (Thermo Fisher Scientific, Inc.) to synthesize complementary DNA, and RT-qPCR was performed with SsoAdvanced ${ }^{\text {TM }}$ Universal SYBR Green Supermix (Bio-Rad Laboratories, Inc., Hercules, CA, USA) according to the manufacturer's protocol. YAP, VEGF and FGF-2 genes were amplified using specific primers and the results were normalized against the human GAPDH gene. A negative control was performed with no RNA added and generated no amplification. The sequences of the primers and the size of the products are listed in Table I. PCR was performed using the CFX Connect ${ }^{\mathrm{TM}}$ Real-Time PCR Detection system (Bio-Rad Laboratories, Inc.). The conditions of the RT-qPCR were as follows: 1 cycle of denaturation at $94^{\circ} \mathrm{C}$ for $5 \mathrm{~min}$, followed by 30 cycles at $94^{\circ} \mathrm{C}$ for $1 \mathrm{~min}, 60^{\circ} \mathrm{C}$ for $1 \mathrm{~min}$ and $72^{\circ} \mathrm{C}$ for $1 \mathrm{~min}$, and a final extension step at $72^{\circ} \mathrm{C}$ for $10 \mathrm{~min}$. Data were normalized using the comparative $\mathrm{Cq}$ method $\left(2^{-\Delta \Delta \mathrm{Cq}}\right)$ (12). A total of 3 parallel RT-qPCR experiments were performed for each group.

Western blotting. To quantitatively determine the protein expression level, western blot analysis was performed. Gastric tumor tissue was cut into small pieces and milled in a mortar with liquid nitrogen. Subsequently, tissue extracts were prepared with radioimmunoprecipitation assay lysis buffer $(100 \mathrm{mM}$ Tris-HCl buffer, $4 \%$ sodium dodecyl sulfate (SDS), 20\% glycerol, $2 \% \beta$-mercaptoethanol; $\mathrm{pH} 6.8$ ). $10 \%$ SDS-polyacrylamide gel electrophoresis was used to separate the samples and the protein bands were transferred onto nitrocellulose membranes. The membranes were blocked with $5 \%$ skim milk for $1 \mathrm{~h}$ at room temperature, followed by hybridization with primary antibodies [YAP, VEGF, FGF-2, TEAD, cyclinD1, GAPDH; dilution, 1:500] at $4^{\circ} \mathrm{C}$ overnight. Following three washes in Tris-buffered saline and Tween 20 (TBST; $150 \mathrm{mM} \mathrm{NaCl}, 0.05 \%$ Tween 20, 50 mM TrisHCl; pH 7.6), the membranes were treated with horseradish peroxidase (HRP)-conjugated goat anti-rabbit secondary antibody 
Table I. Sequences of primers.

Primer

Sequence

Size of product, bp

$\begin{array}{ll}\text { YAP forward } & \text { CCTGATGGATGGGAACAAGC } \\ \text { YAP reverse } & \text { GCACTCTGACTGATTCTCTGG } \\ \text { VEGF forward } & \text { GCTTACTCTCACCTGCTTCTG } \\ \text { VEGF reverse } & \text { GGCTGCTTCTTCCAACAATG } \\ \text { FGF-2 forward } & \text { ATCAAAGGAGTGTGTGCTAACC } \\ \text { FGF-2 reverse } & \text { ACTGCCCAGTTCGTTCAGTG } \\ \text { GAPDH forward } & \text { TGACTTCAACAGCGACACCCA } \\ \text { GAPDH reverse } & \text { CACCCTGTTGCTGTAGCCAAA }\end{array}$

YAP, yes-associated protein; VEGF, vascular endothelial growth factor; FGF-2, fibroblast growth factor 2; GAPDH, glyceraldehyde-3-phosphate dehydrogenase.

(dilution, 1:3,000; ab205718; Abcam, Cambridge, UK) for $2 \mathrm{~h}$ at room temperature. Subsequently, the membranes were washed with TBST three times and the immunoreactive bands were visualized using the ECL Western Blotting Substrate (Thermo Fisher Scientific, Inc.) according to the manufacturer's protocol. The relative protein expression in various cell lines was normalized to GAPDH expression levels. The experiments were performed three times.

Hematoxylin and eosin $(H \& E)$ staining. To study tumor morphology, H\&E staining was used. Briefly, tumor samples were fixed with paraformaldehyde and embedded with paraffin. The paraffin blocks were sliced into $5-\mu \mathrm{m}$ thick sections and mounted onto glass microscope slides. Subsequently, the slides were deparaffinized using xylene and graded alcohols prior to being stained with H\&E. A total of 5 randomly selected microscopic fields from each slide were examined by two pathologists with no prior information about the samples using a microscope (BA410; Motic Incoporation, Ltd., Causeway Bay, Hong Kong).

Immunohistochemistry (IHC). Tumor specimens were prepared as described above. Subsequently, the tissue sections were deparaffinized and placed in antigen retrieval solution (Abcam) for $15 \mathrm{~min}$ at $100^{\circ} \mathrm{C}$. Following incubation in $1 \%$ bovine serum albumin (Sigma-Aldrich) for $30 \mathrm{~min}$, primary antibodies [YAP, CD31, cyclinA, cyclinD1, cyclinE; dilution, 1:200] were applied to the slides for $2 \mathrm{~h}$ at $37^{\circ} \mathrm{C}$. Following washing with PBS, the sections were additionally incubated with the HRP-conjugated donkey anti-goat secondary antibody (dilution, 1:2,000; ab205723; Abcam) and avidin-conjugated horseradish peroxidase (43-4423; Thermo Fisher Scientific, Inc.). Finally, the sections were treated with 3,3'-diaminobenzidine, and counterstained with hematoxylin, and dehydrated. A negative control was performed by replacement of primary antibody with PBS. Two pathologists reviewed the results, with IHC staining intensity determined by Image-Pro Plus 6.0 (Media Cybernetics, Inc., Rockville, MD, USA).

Proliferation assay and apoptosis assay. The cell proliferation assay procedure was performed in same manner as the aforementioned IHC procedure, except anti-Ki-67 primary

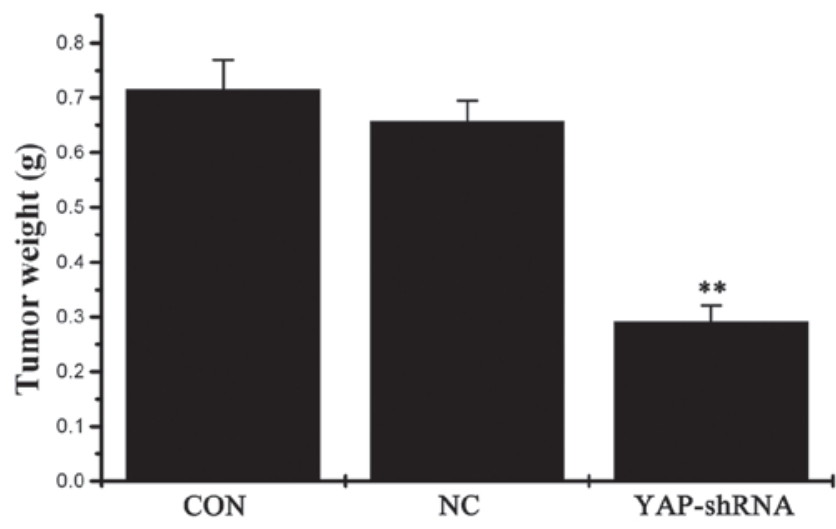

Figure 1. The weight of orthotopic gastric cancer in the three groups. Data are presented as the mean \pm standard deviation. ${ }^{* *}$ The mean of this group had significant difference with the mean of $\mathrm{CON}$ group $(\mathrm{P}<0.01)$ and the mean of this group also had significant difference with the mean of $\mathrm{NC}$ group $(\mathrm{P}<0.01)$. CON, untransfected cells; $\mathrm{NC}$, negative control vector-transfected cells; YAP-shRNA, yes-associated protein small hairpin RNA vector-transfected cells.

antibody was used to measure cancer cell proliferation ability. Terminal deoxynucleotidyl transferase dUTP nick end labeling (TUNEL) assay was used to measure the cancer cell apoptosis rate, and was performed according to manufacturer's protocol. The proliferation rate and apoptotic rate were determined quantitatively by counting the number of positively stained cells in 5 fields at magnification, x200.

Statistical analysis. Statistically significant differences between groups were determined with SPSS version 15.0 (SPSS, Inc., Chicago, IL, USA). The differences between each group were tested for significance using the Student's two-sided t-test. $\mathrm{P}<0.05$ was considered to indicate a statistically significant difference.

\section{Results}

Silencing of the YAP gene inhibits orthotopic gastric tumor growth. The present study analyzed the role of YAP-shRNA in tumor growth in vivo. As shown in Fig. 1, the average tumor weight was significantly reduced in the YAP-shRNA group 
Table II. Cases of gastric cancer metastasis and ascites in severe combined immunodeficiency mice following orthotopic implantation.

\begin{tabular}{lcccccr}
\hline & & \multicolumn{3}{c}{ Metastasis } & Mesentery & Ascites \\
\cline { 3 - 6 } Group & $\mathrm{n}$ & Liver & Diaphragm & Lymph node & 7 & 6 \\
\hline CON & 8 & 6 & 3 & 4 & 6 & 5 \\
NC & 8 & 4 & 2 & 5 & 2 & 1 \\
YAP-shRNA & 8 & 0 & 0 & 1 & & 7 \\
\hline
\end{tabular}

CON, untransfectedcells; NC, negative control vector-transfected cells; YAP-shRNA,yes-associated protein small hairpinRNAvector-transfected cells.

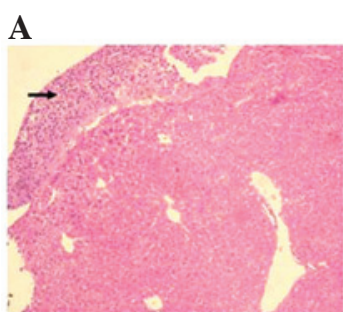

$\mathbf{E}$

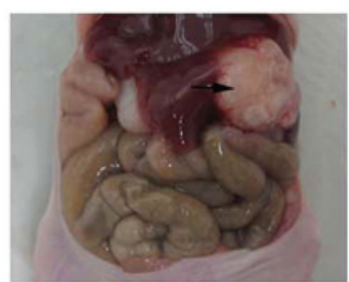

B

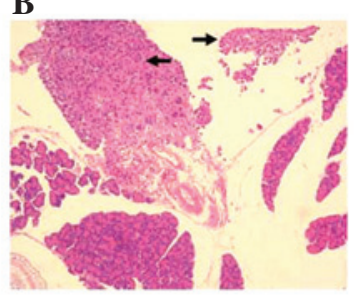

$\mathbf{F}$

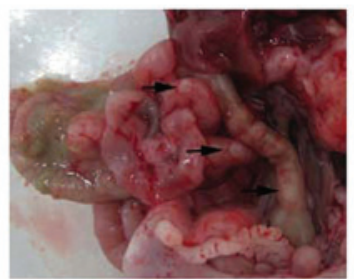

C

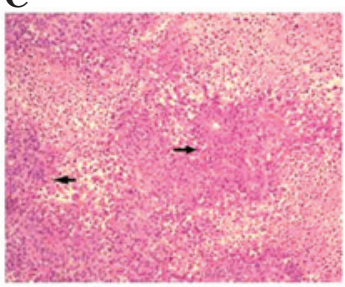

G

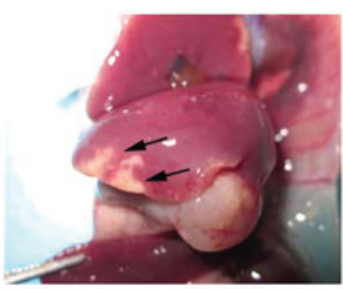

D

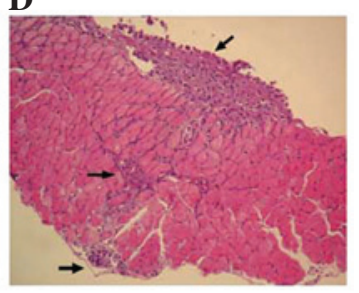

$\mathbf{H}$

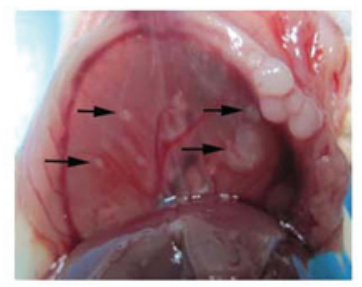

Figure 2. Severe combined immunodeficiency mouse metastatic tumors. (A) HE staining of liver metastasis; (B) HE staining of diaphragm metastasis; (C) HE staining of lymph node metastasis; (D) HE staining of mesentery metastasis (all magnification, x200); (E) Liver metastasis; (F) Diaphragm metastasis; (G) Mesentery metastasis; (H) Orthotopic gastric cancer; HE, hematoxylin and eosin.

A

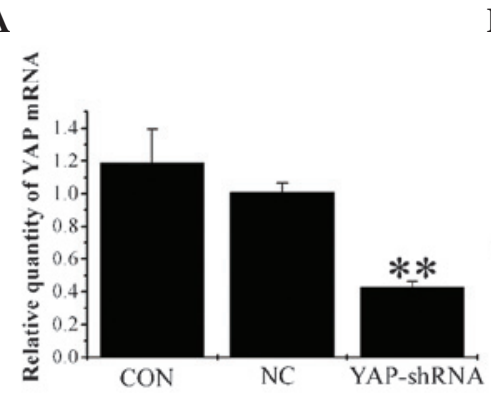

D

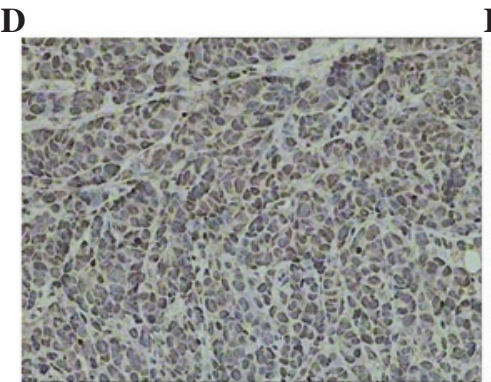

B

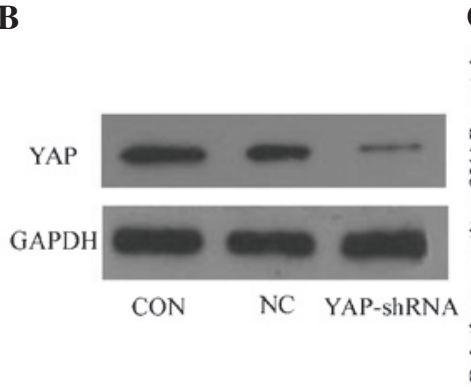

$\mathbf{E}$

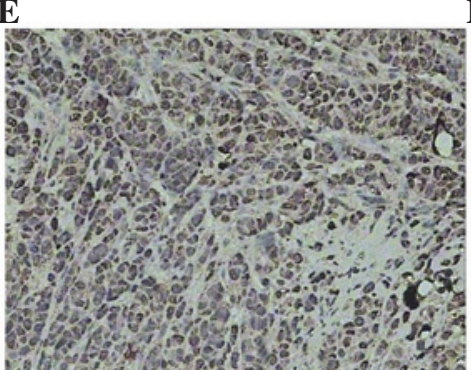

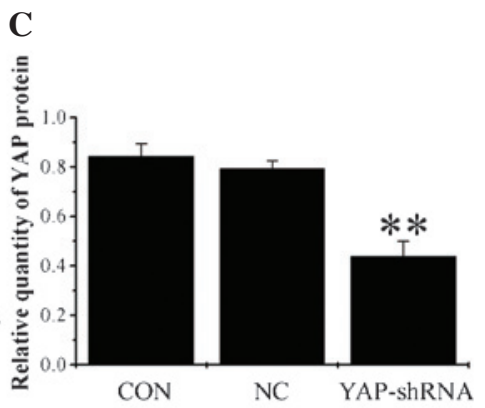

F

Figure 3. Expression of yes-associated protein in the three orthotopic gastric cancer groups. (A) Reverse transcription-polymerase chain reaction analysis of YAP mRNA level. (B) Western blot analysis of YAP protein expression level and (C) quantification of western blot analysis. YAP expression levels were detected by immunohistochemistry in (D) CON group, (E) NC group and (F) YAP-shRNA group (all magnification, x200). Data are presented as the mean \pm standard deviation. ${ }^{* *} \mathrm{P}<0.01$ vs. CON and NC groups. CON, untransfected cells; NC, negative control vector-transfected cells; YAP-shRNA, yes-associated protein small hairpin RNA vector-transfected cells; mRNA, messenger RNA; GAPDH, glyceraldehyde-3-phosphate dehydrogenase. 
$\mathbf{A}$
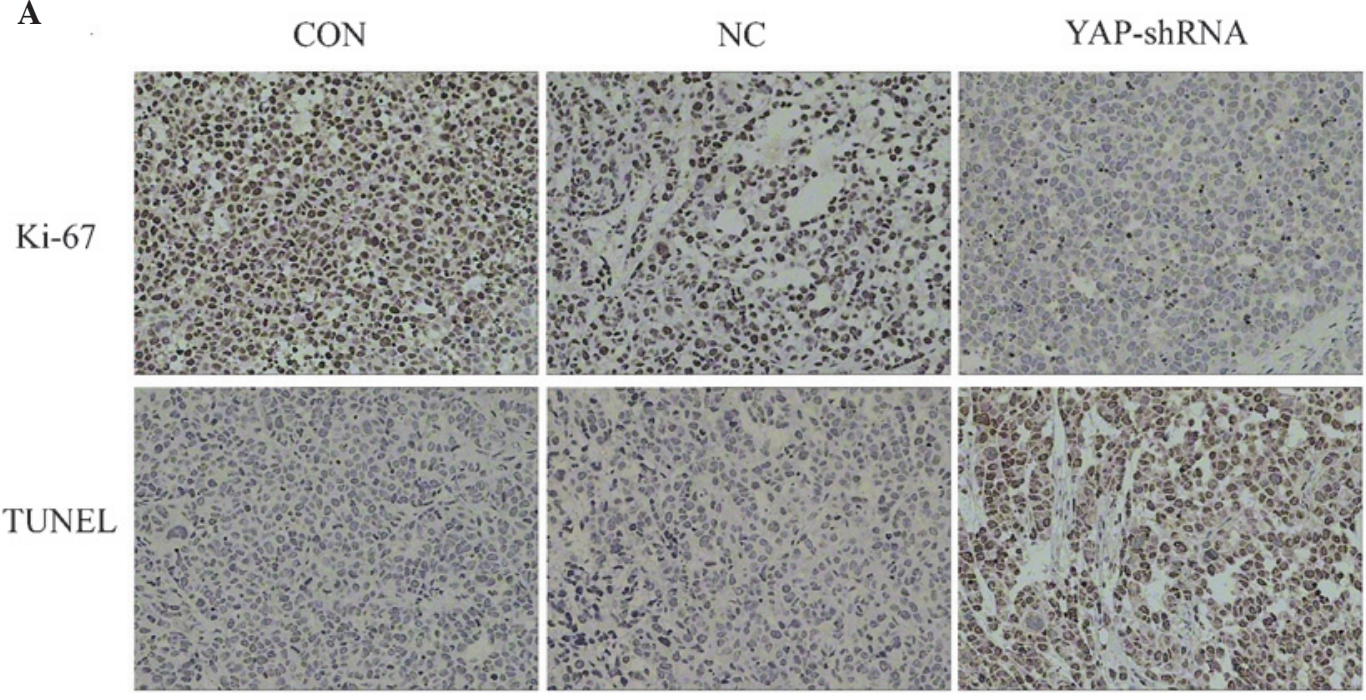

B
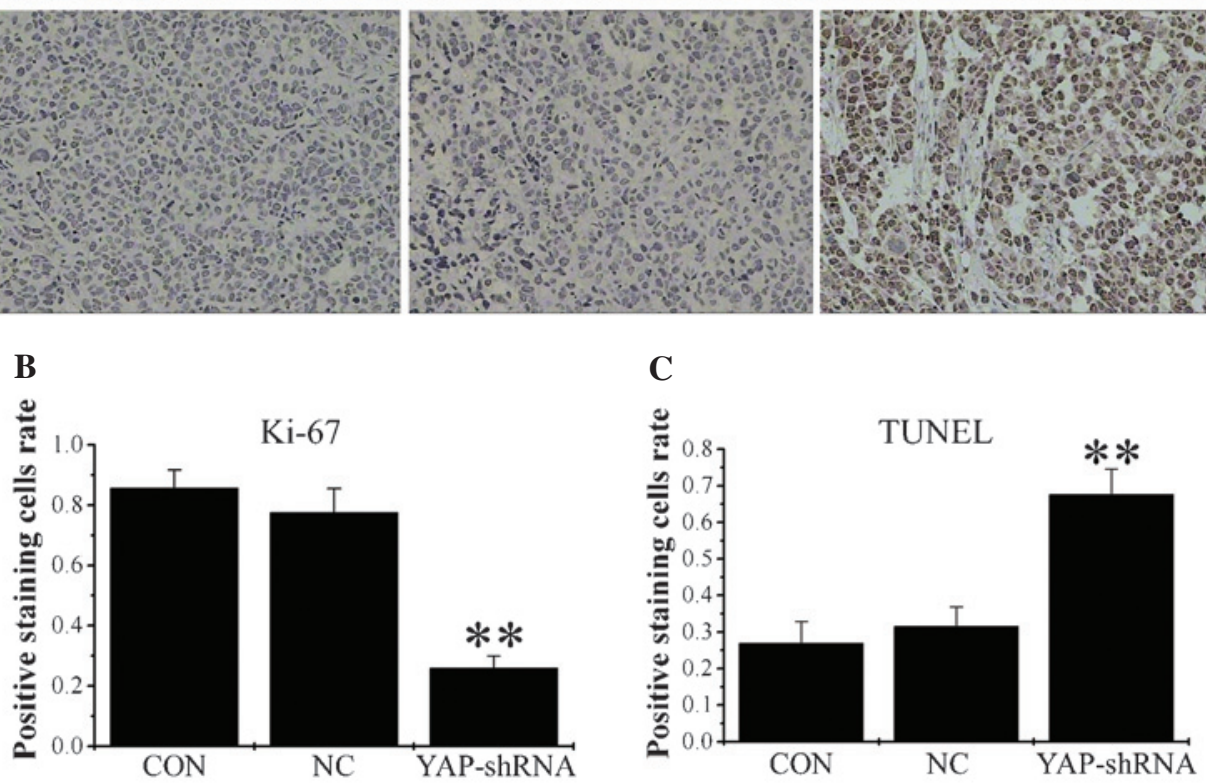

Figure 4. Silencing of YAP gene expression inhibits gastric cancer cell proliferation and promotes apoptosis. (A, upper panels) and (B) Ki-67 expression levels were detected by immunohistochemistry. (A, lower panels) and (C) TUNEL analysis of cell apoptotic rate. Magnification, x200. Data are presented as the mean \pm standard deviation. ${ }^{* *} \mathrm{P}<0.01$ vs. CON and NC groups. CON, untransfected cells; NC, negative control vector-transfected cells; YAP-shRNA, yes-associated protein small hairpin RNA vector-transfected cells; TUNEL, terminal deoxynucleotidyl transferase dUTP nick end labeling.

compared with the NC and CON groups (YAP-shRNA, $0.292 \pm 0.029 \mathrm{~g}$ vs. NC, $0.657 \pm 0.038 \mathrm{~g} ; \mathrm{P}<0.01$; YAP-shRNA, $0.292 \pm 0.029 \mathrm{~g}$ vs. CON, $0.715 \pm 0.054 \mathrm{~g} ; \mathrm{P}<0.01)$. No marked difference was observed between the NC group and the CON group. It was concluded that silencing of the YAP gene in SGC7901 cells may inhibit orthotopic tumor growth (Fig. 1).

Silencing of the YAP gene reduces metastatic tumor formation. To elucidate the effect of YAP knockdown on gastric cancer metastasis, a gastric cancer orthotopic implantation model was constructed. A total of two months later, all mice were sacrificed due to severe cachexia. Tumors from the orthotopic implantation and metastasis in other organs were dissected and examined by H\&E staining (Fig. 2). Histological analysis of tumor metastases revealed the typical structure of metastatic adenocarcinoma. Table II summarizes the differences between distant metastasis in the three groups. The incidence of liver, diaphragm, lymph node and mesentery metastasis, as well as ascites in the NC group was 75, 37.5, 50, 87.5 and $75 \%$, respectively. In the CON group, the incidence of liver, diaphragm, lymph node and mesentery metastasis, as well as ascites was $50,25,62.5,75$ and $62.5 \%$, respectively. By contrast, in the YAP-shRNA group, $12.5 \%$ had lymph node metastasis, $25 \%$ had mesentery metastasis and $12.5 \%$ had ascites. No detectable tumors in the liver and diaphragm were identified. These results indicated that YAP knockdown inhibited tumor metastasis in the gastric orthotopic implantation mouse model.

YAP-shRNA reduces YAP expression in orthotopic implantation models. The stability of YAP-shRNA in orthotopic gastric tumors was confirmed by RT-qPCR and western blotting. As shown in Fig. 3A, B and C, a marked inhibition of YAP mRNA and protein expression was observed in the YAP-shRNA group compared with the NC and CON groups (mRNA YAP-shRNA, $0.425 \pm 0.04$ vs. NC, $1.01 \pm 0.056 ; \mathrm{P}<0.01$; YAP-shRNA, $0.425 \pm 0.04$ vs. $\mathrm{CON}, 1.186 \pm 0.21 ; \mathrm{P}<0.01$; protein YAP-shRNA, $0.44 \pm 0.059$ vs. NC, 0.794 \pm 0.033 ; $\mathrm{P}<0.01$; YAP-shRNA, $0.44 \pm 0.059$ vs. $\mathrm{CON}, 0.845 \pm 0.049 ; \mathrm{P}<0.01)$. Furthermore, following IHC, strong staining for YAP was observed in the NC and CON groups, whereas in the YAP-shRNA group, the tissues displayed only moderate staining (Fig. 3D, E and F). Therefore, this confirmed that the efficiency of YAP-shRNA silencing is not altered in vivo compared with its performance in vitro in cell lines.

Silencing of YAP reduces proliferation and promotes apoptosis. To examine proliferative ability, sections from orthotopic gastric tumor were stained with anti-Ki-67 antibody, and the percentage of cells positive for Ki-67 expression was calculated. The results of the present study demonstrated that there were fewer positive cells in the YAP-shRNA group compared 


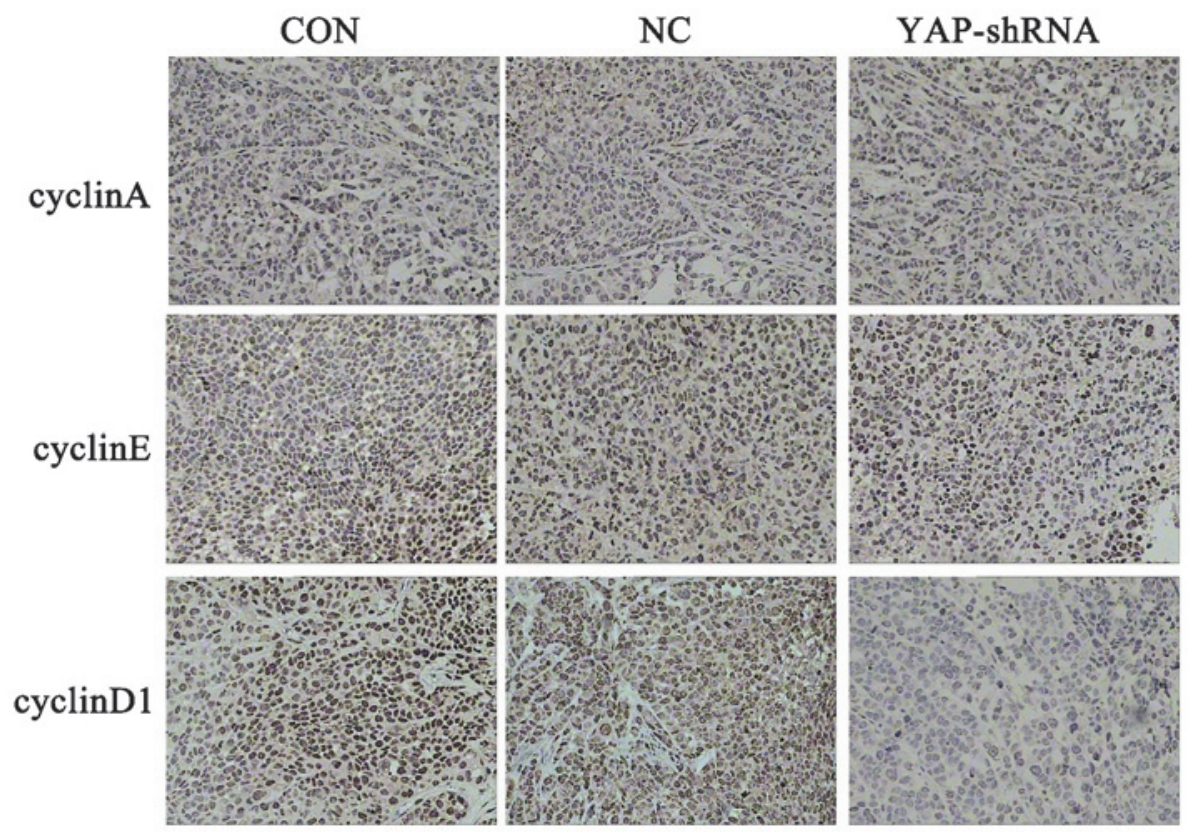

Figure 5. Expression of cyclinA, cyclinE, cyclinD1 in orthotopic implantation gastric tumors was detected by immunohistochemical staining (magnification, x200). CON, untransfected cells; NC, negative control vector-transfected cells; YAP-shRNA, yes-associated protein small hairpin RNA vector-transfected cells.

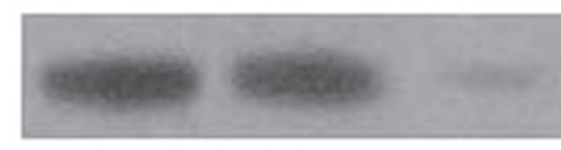

YAP

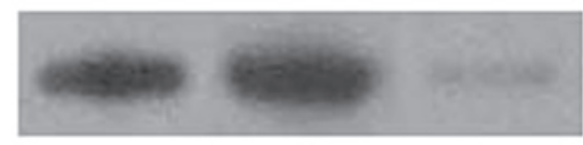

TEAD

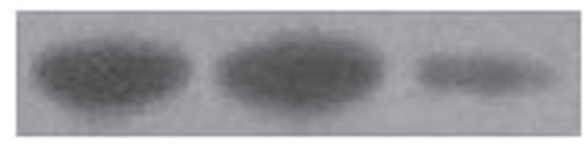

CyclinD1

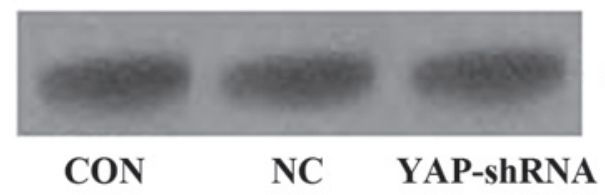

GAPDH

Figure 6. Expression levels of YAP, TEAD and cyclinD1 in orthotopic implantation gastric tumors were detected by western blotting. CON, untransfected cells; NC, negative control vector-transfected cells; YAP-shRNA, yes-associated protein small hairpin RNA vector-transfected cells; TEAD, TEA domain family member 1; GAPDH, glyceraldehyde-3-phosphate dehydrogenase.

with the other two groups (YAP-shRNA, $0.259 \pm 0.04$ vs. NC, $0.775 \pm 0.08 ; \mathrm{P}<0.01$; YAP-shRNA, $0.259 \pm 0.04$ vs. CON, $0.875 \pm 0.06 ; \mathrm{P}<0.01)$. No significant differences were observed between the $\mathrm{NC}$ and $\mathrm{CON}$ groups (Fig. 4). As Ki-67 is a widely used proliferation biomarker and its expression indicates active cell growth, these findings demonstrate that YAP-shRNA reduced cancer cell proliferation in the mouse gastric cancer model (13).

To analyze tumor cell apoptosis, the tumor sections from orthotopic gastric tumor were stained for apoptotic markers using a TUNEL staining assay. The number of apoptotic cells was significantly increased in the YAP-shRNA group compared with the other two groups (YAP-shRNA, $0.676 \pm 0.07$ vs. NC, $0.315 \pm 0.05 ; \mathrm{P}<0.01 ;$ YAP-shRNA, $0.676 \pm 0.07$ vs. CON, $0.269 \pm 0.06 ; \mathrm{P}<0.01)$. No significant difference was observed between the NC and CON groups (Fig. 4). Therefore, YAP-shRNA promotes gastric cancer cell apoptosis in the mouse gastric cancer model.

Silencing of the YAP gene affects the expression of cyclinD1. A previous study by the present authors revealed that cells are arrested in G1 phase in the YAP-shRNA group in vitro (11). Therefore, the present study examined the expression of cyclin A, cyclinD1 and cyclinE in the orthotopic implantation gastric tumor by immunohistochemical assay. As shown in Fig. 5, tumor cells transfected with YAP-shRNA demonstrated lower expression of cyclinD1 compared with that the $\mathrm{NC}$ and CON groups, while no significant difference in expression was observed for cyclinA and cyclinE.

CycinD1 is a regulatory factor that regulates cell cycle transition from G0 to G1 (14). Our previous in vitro results are consistent with our in vivo results (10). It is well-known that YAP has a significant role in cancer cell proliferation and is an important transcription factor for regulating the cell cycle (15). A previous study demonstrated that YAP regulates cyclinD1 transcription directly in cooperation with TEAD in malignant mesothelioma cells (16). However, to the best of our knowledge, no such study has been performed for gastric cancer; therefore, the present study examined the effect of knockdown of YAP on the expression of TEAD and cyclinD1 in vivo. As shown in Fig. 6, the expression of these factors was significantly decreased when YAP gene expression was silenced, which indicates that YAP/TEAD/cyclinD1 signaling may be involved in YAP silencing-induced growth inhibition of gastric cancer. 
A

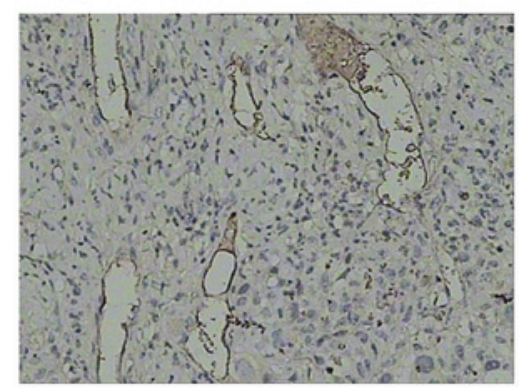

B

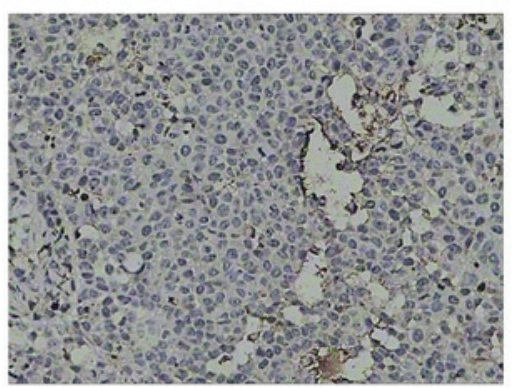

C

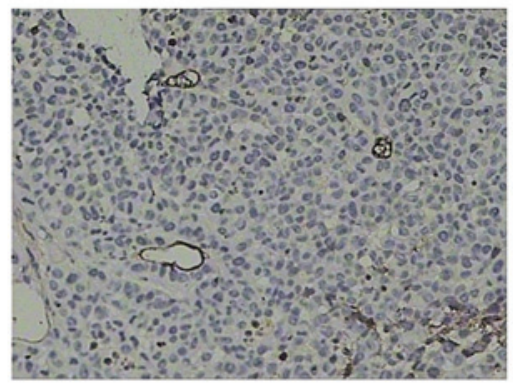

D

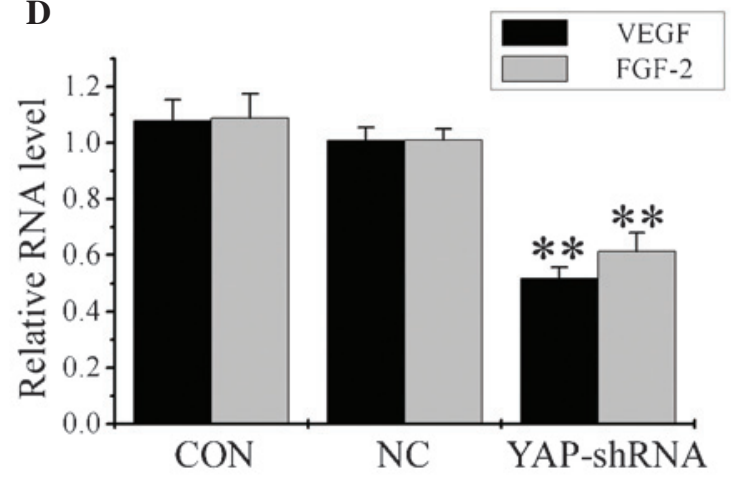

F

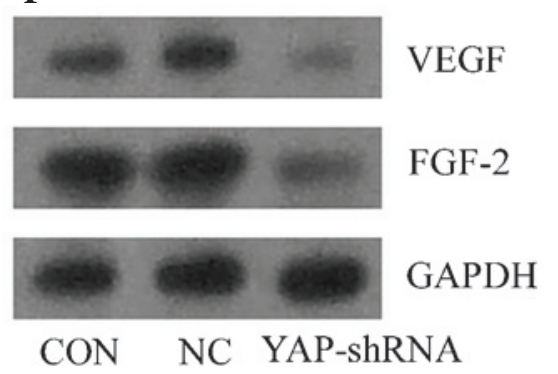

E
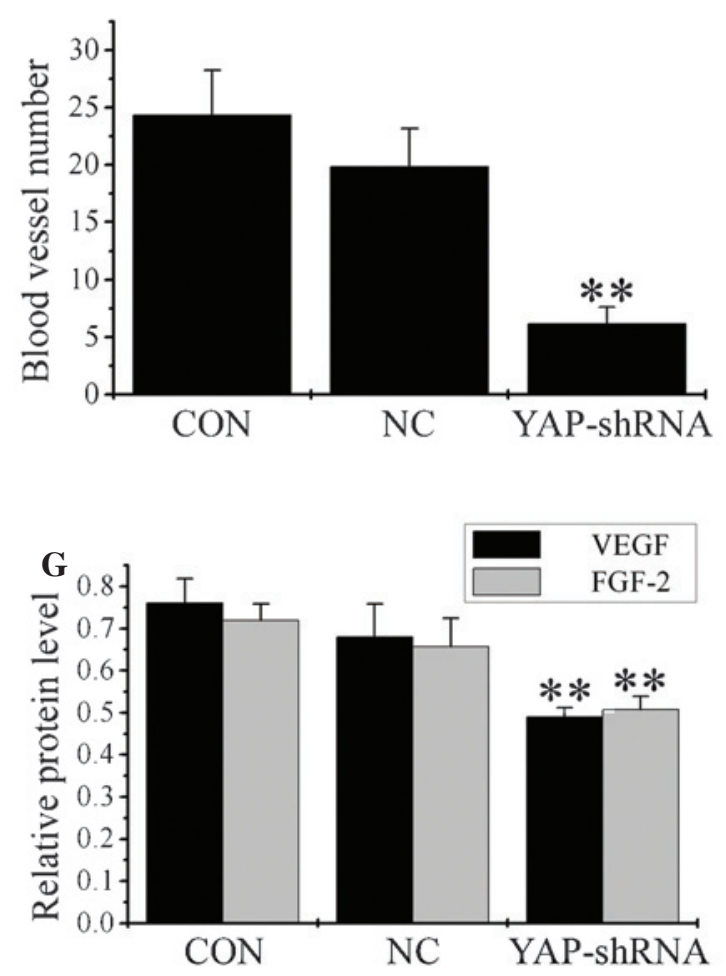

Figure 7. Inhibition of YAP expression suppresses angiogenesis in orthotopic implantation gastric cancer. CD31 staining images in (A) CON group, (B) NC group and (C) YAP-shRNA group (all magnification, x200). (D) Reverse transcription-polymerase chain reaction analysis of VEGF and FGF-2 messenger RNA level. (E) Mean number of blood vessels identified through CD31 staining. (F) Western blot analysis of VEGF and FGF-2 protein level and (G) quantification of the western blot. Data are presented as the mean \pm standard deviation. ${ }^{* *} \mathrm{P}<0.01 \mathrm{vs}$. CON and NC groups. CON, untransfected cells; NC, negative control vector-transfected cells; YAP-shRNA, yes-associated protein small hairpin RNA vector-transfected cells; VEGF, vascular endothelial growth factor; FGF-2, fibroblast growth factor 2; GAPDH, glyceraldehyde-3-phosphate dehydrogenase.

Silencing of the YAP gene inhibits angiogenesis, as well as $V E G F$ and FGF-2 expression in vivo. To quantify the in vivo angiogenesis ability, sections of orthotopic gastric cancer from each group were stained for endothelial cell specific CD31. The number of vessels within each section was quantified in five different fields at magnification, $\mathrm{x} 200$. Tumors derived from the YAP-shRNA group had reduced intratumoral microvessel density compared with the NC and CON groups (YAP-shRNA, $6.167 \pm 1.47$ vs. NC, $19.83 \pm 3.31 ; \mathrm{P}<0.01$; YAP-shRNA, $6.167 \pm 1.47$ vs. CON, $24.33 \pm 3.93$; P $<0.01$; Fig. $7 \mathrm{~A}, \mathrm{~B}, \mathrm{C}$ and D).

VEGF and FGF-2 are potent mitogens for vascular endothelial cells and have a significant role in gastric cancer neovascularization (17). To additionally explore the effects of YAP downregulation on angiogenesis in vivo, the present study examined the expression of VEGF and FGF-2. RT-qPCR and western blotting revealed that YAP-shRNA-transfected cells constitutively expressed low levels of VEGF and FGF-2 compared with the $\mathrm{NC}$ and $\mathrm{CON}$ groups (Fig. 7E, F and G). Infiltration of blood vessels is a sign of hematogenous metastasis (17). These results indicate that YAP expression affects gastric cancer angiogenesis in vivo.

\section{Discussion}

The Hippo signaling pathway comprises a series of cytoplasmic tumor suppressor proteins, including merlin, large tumor suppressor kinase $1 / 2$ and macrophage stimulating $1 / 2$, and is thought to play a critical role in determining the size of organs and tissues (15). When activated, the Hippo signaling pathway maintains the transcriptional activator YAP in phosphorylated 
form in the cytoplasm and prevents cell proliferation (18). When the Hippo signaling pathway is blocked, YAP is translocated to the nucleus and induces the expression of a variety of proteins that are associated with a malignant cell phenotype (18).

YAP is the central player within the Hippo signaling pathway, which has been considered an effective target for cancer therapy (19). The role of YAP in oncogenesis has gained significant attention. Increasing evidence has demonstrated that YAP is involved in the development and progression of cancer (15). However, whether YAP is associated with cancer metastasis remains unclear. In our previous studies, it was demonstrated that targeting the YAP gene using RNAi inhibited the migration, invasion, anchorage-independent growth and angiogenesis ability of SGC-7901 cells $(10,11)$. Furthermore, in our previous study, a lentivirus plasmid vector expressing shRNAs directed against the YAP gene was developed, and it was demonstrated that RNAi driven by these shRNA-based lentiviruses was able to effectively silence YAP gene expression at the mRNA and protein level in vitro (11). Furthermore, functional analyses revealed that abrogation of YAP expression not only suppressed growth and induced apoptosis of cancer cells, but additionally inhibited cancer cell metastasis in vitro (11). These studies indicated that YAP may be important for cancer metastasis at a cellular level. Therefore, in the present study a tumor model was developed in SCID mice to further study the YAP gene.

The present study demonstrated that silencing of YAP caused an inhibition of gastric cancer growth in an orthotopic gastric cancer model. A surgical orthotopic implantation model involves xenotransplantation of fresh solid tumor into immunodeficient mice $(20,21)$, and this model was utilized in the present experiments. Silencing of YAP is associated with a significant reduction of the incidence of metastasis and ascites. For gastric cancer patients, metastasis is an indicator of poor prognosis (22). Silencing of YAP is associated with decreased cell migration and invasion (Table II and Fig. 7). This suggests that YAP not only regulates gastric cancer cell proliferation, but additionally has an impact on cancer metastasis.

Apoptosis is an important mechanism for elimination of potentially tumorigenic cells (23). Quantification of apoptotic cells by TUNEL assay was performed in vivo. Experimental data demonstrated that apoptosis of cells transfected with YAP-shRNA vectors was significantly increased compared with control groups. The effect on cancer cell proliferation was investigated by Ki-67 assay, which is the most widely used proliferation biomarker (24). It was demonstrated that YAP downregulation reduces the expression levels of Ki-67. Therefore, it appears reasonable to hypothesize that the YAP gene may promote proliferation and inhibit apoptosis in gastric cancer.

Cell cycle factors have a significant role in cell cycle progression (25). The present study additionally examined the expression of cell cycle associated proteins cyclinA, cyclinD1 and cyclinE in the three groups following YAP inhibition. It was observed that the expression level of cyclinD1 is significantly decreased when YAP expression is silenced, while the expression of cyclinA and cyclinE is not significantly changed. CyclinD1 has a significant role in regulating cell cycle progression (14). CyclinD1 mRNA and protein are overexpressed in several types of human cancer (26).
YAP is not able to bind the cyclinD1 gene directly, as it possesses no DNA binding sites; YAP activates its targets by binding transcription factors (27). Consistent with previous results, YAP and TEAD promoted cyclinD1 expression in gastric cancer (16).

Increased angiogenesis provides a route of dissemination from the primary tumor and may contribute to the growth of the metastatic tumor (28). Therefore, primary tumors that are more heavily vascularized are more aggressive and demonstrate increased metastatic potential (29). Antitumor efficacy of anti-angiogenic drugs could be judged by reduced intratumoral microvessel density (30). Although few studies have demonstrated the association between YAP expression and angiogenesis, the results of the present study demonstrated a significant downregulation of tumor capillary number, as well as VEGF and FGF-2 expression levels, following stable YAP gene silencing.

Previous studies demonstrated that silencing of the YAP gene inhibited gastric cancer cell proliferation, migration and invasion in vitro $(10,11)$. The results of the present study demonstrate that knockdown of the YAP gene significantly reduces tumor growth and suppresses cancer metastasis to other organs in vivo in the orthotopic gastric cancer models. The mechanism of this anti-tumor effect appears to include activation of cancer cell apoptosis, and inhibition of cancer cell proliferation and migration. Therefore, the anti-tumor effects of YAP-shRNA on gastric cancer are not only limited to exist in vitro, but also exist in vivo. Thus, the results of the present study suggest that YAP may be a potential molecular target for gastric cancer therapy.

In conclusion, the present study indicated that YAP may exhibit an important role in the metastasis of gastric cancer, and that downregulation of YAP gene expression may present a useful therapeutic modality for gastric cancer treatment.

\section{References}

1.PDQ Screening and Prevention Editorial Board: Stomach (Gastric) Cancer Prevention (PDQ): Health Professional Version. http://www.cancer. gov/types/stomach/hp/stomach-prevention-pdq. Accessed February 5, 2016

2. Guo HQ, Guan P, Shi HL, Zhang X, Zhou BS and Yuan Y: Prospective cohort study of comprehensive prevention to gastric cancer. World J Gastroenterol 9: 432-436, 2003.

3. Guo $X$ and Zhao B: Integration of mechanical and chemical signals by YAP and TAZ transcription coactivators. Cell Biosci 3: 33, 2013.

4. Hong W: Angiomotin'g YAP into the nucleus for cell proliferation and cancer development. Sci Signal 6: pe27, 2013.

5. Overholtzer M, Zhang J, Smolen GA, Muir B, Li W, Sgroi DC, Deng CX, Brugge JS and Haber DA: Transforming properties of YAP, a candidate oncogene on the chromosome 11q22 amplicon. Proc Natl Acad Sci USA 103: 12405-12410, 2006.

6. Xiao W, Wang J, Ou C, Zhang Y, Ma L, Weng W, Pan Q and Sun F: Mutual interaction between YAP and c-Myc is critical for carcinogenesis in liver cancer. Biochem Biophys Res Commun 439: 167-172, 2013.

7. Liu-Chittenden Y, Huang B, Shim JS, Chen Q, Lee SJ, Anders RA, Liu JO and Pan D: Genetic and pharmacological disruption of the TEAD-YAP complex suppresses the oncogenic activity of YAP. Genes Dev 26: 1300-1305, 2012.

8. Felley-Bosco E and Stahel R: Hippo/YAP pathway for targeted therapy. Transl Lung Cancer Res 3: 75-83, 2014.

9. Okamoto K and Murawaki Y: The therapeutic potential of RNA interference: Novel approaches for cancer treatment. Curr Pharm Biotechnol 13: 2235-2247, 2012. 
10. Zhou Z, Zhu JS and Xu ZP: RNA interference mediated YAP gene silencing inhibits invasion and metastasis of human gastric cancer cell line SGC-7901. Hepatogastroenterology 58: 2156-2161, 2011.

11. Zhou Z, Zhu JS, Xu ZP and Zhang Q: Lentiviral vector-mediated siRNA knockdown of the YAP gene inhibits growth and induces apoptosis in the SGC7901 gastric cancer cell line. Mol Med Rep 4: 1075-1082, 2011.

12. Livak KJ and Schmittgen TD: Analysis of relative gene expression data using real-time quantitative PCR and the 2(-Delta Delta C(T)) method. Methods 25: 402-408, 2001.

13. Li LT, Jiang G, Chen Q and Zheng JN: Ki67 is a promising molecular target in the diagnosis of cancer (review). Mol Med Rep 11:1566-1572, 2015.

14. Pestell RG: New roles of cyclin D1. Am J Pathol 183:3-9, 2013.

15. Moroishi T, Hansen CG and Guan KL: The emerging roles of YAP and TAZ in cancer. Nat Rev Cancer 15: 73-79, 2015.

16. Mizuno T, Murakami H, Fujii M, Ishiguro F, Tanaka I, Kondo Y, Akatsuka S, Toyokuni S, Yokoi K, Osada H and Sekido Y: YAP induces malignant mesothelioma cell proliferation by upregulating transcription of cell cycle-promoting genes. Oncogene 31: 5117-5122, 2012.

17. Cao R, Ji H, Feng N, Zhang Y, Yang X, Andersson P, Sun Y, Tritsaris K, Hansen AJ, Dissing S and Cao Y: Collaborative interplay between FGF-2 and VEGF-C promotes lymphangiogenesis and metastasis. Proc Natl Acad Sci USA 109: 15894-15899, 2012.

18. Hong W and Guan KL: The YAP and TAZ transcription co-activators: Key downstream effectors of the mammalian Hippo pathway. Semin Cell Dev Biol 23: 785-793, 2012.

19. Kang W, Tong JH, Chan AW, Lee TL, Lung RW, Leung PP, So KK, Wu K, Fan D, Yu J, et al: Yes-associated protein 1 exhibits oncogenic property in gastric cancer and its nuclear accumulation associates with poor prognosis. Clin Cancer Res 17: 2130-2139, 2011.

20. Bai F, Guo X, Yang L, Wang J, Shi Y, Zhang F, Zhai H, Lu Y, Xie H, $\mathrm{Wu} \mathrm{K}$ and Fan D: Establishment and characterization of a high metastatic potential in the peritoneum for human gastric cancer by orthotopic tumor cell implantation. Dig Dis Sci 52: 1571-1578, 2007.
21. Thalheimer A, Otto C, Bueter M, Illert B, Gattenlohner S, Gasser M, Fein M, Germer CT and Waaga-Gasser AM: Tumor cell dissemination in a human colon cancer animal model: Orthotopic implantation or intraportal injection? Eur Surg Res 42: 195-200, 2009.

22. Lehnert T, Rudek B, Buhl K and Golling M: Surgical therapy for loco-regional recurrence and distant metastasis of gastric cancer. Eur J Surg Oncol 28: 455-461, 2002.

23. Jamali $M$ and Chetty R: Predicting prognosis in gastroentero-pancreatic neuroendocrine tumors: An overview and the value of Ki-67 immunostaining. Endocr Pathol 19: 282-288, 2008

24. Duchrow M, Schlüter C, Key G, Kubbutat MH, Wohlenberg C, Flad HD and Gerdes J: Cell proliferation-associated nuclear antigen defined by antibody Ki-67: A new kind of cell cycle-maintaining proteins. Arch Immunol Ther Exp (Warsz) 43: 117-121, 1995.

25. Amoedo ND, El-Bacha T, Rodrigues MF and Rumjanek FD Cell cycle and energy metabolism in tumor cells: Strategies for drug therapy. Recent Pat Anticancer Drug Discov 6: 15-25, 2011.

26. Umekita Y, Ohi Y, Sagara Y and Yoshida H: Overexpression of cyclinD1 predicts for poor prognosis in estrogen receptor-negative breast cancer patients. Int J Cancer 98: 415-418, 2002

27. Lamar JM, Stern P, Liu H, Schindler JW, Jiang ZG and Hynes RO: The Hippo pathway target, YAP, promotes metastasis through its TEAD-interaction domain. Proc Nat Acad Sci USA 109: E2441-E2450, 2012.

28. Singh S, Sadanandam A and Singh RK: Chemokines in tumor angiogenesis and metastasis. Cancer Metastasis Rev 26: 453-467, 2007.

29. Barzi A and Thara E: Angiogenesis in esophageal and gastric cancer: a paradigm shift in treatment. Expert Opin Biol Ther 14: 1319-1332, 2014.

30. Tuettenberg J, Friedel C and Vajkoczy P: Angiogenesis in malignant glioma - a target for antitumor therapy? Crit Rev Oncol Hematol 59: 181-193, 2006. 